\title{
A Wide QRS Complex, Long RP Tachycardia: What is the Tachycardia Mechanism?
}

\author{
FRED MORADY, M.D. \\ From the Cardiology Division, Department of Internal Medicine, University of Michigan Medical Center, Ann Arbor, Michigan
}

\section{Case Presentation}

A 78-year-old man underwent an electrophysiologic procedure because of a 25 -year history of recurrent episodes of paroxysmal tachycardia. A wide QRS complex tachycardia that had a long $\mathrm{RP}$ interval was induced by ventricular pacing (Fig. $1)$. The cycle length of the tachycardia was 540 msec. An atrial extrastimulus introduced in the high right atrium during the first half of diastole terminated the tachycardia (Fig. 2). The tachycardia also was terminated by ventricular pacing at a cycle length of $340 \mathrm{msec}$ (Fig. 3). What is the tachycardia mechanism?

\section{Commentary}

The 12-lead ECG in Figure 1 demonstrates that the QRS complexes during the tachycardia have a right bundle branch block configuration and a duration of $180 \mathrm{msec}$. Furthermore, the QRS complexes in the precordial leads are concordant. These findings are typical of a ventricular tachycardia arising in the left ventricle, or of ventricular activation over a left-sided accessory AV pathway. There is one $\mathrm{P}$ wave preceding each QRS complex, and the $\mathrm{P}$ waves are inverted in the inferior leads, compatible with ventricular tachycardia and a 1:1 pattern of slow VA conduction, antidromic reciprocating tachycardia, or an atrial tachycardia with 1:1 AV conduction over an accessory path-

J Cardiovasc Electrophysiol, Vol. 8, pp. 1204-1206, October 1997.

Address for correspondence: Fred Morady, M.D., University of Michigan Medical Center, 1500 East Medical Center Drive, B1F245, Ann Arbor, MI 48109-0022. Fax: 313-936-7026.

Manuscript received 27 February 1997; Accepted for publication 27 February 1997. way. Given the QRS duration of $180 \mathrm{msec}$, the right bundle branch block morphology, and the concordant pattern of QRS complexes in the precordial leads, other mechanisms of wide QRS tachycardia, such as paroxysmal supraventricular tachycardia with underlying or rate-related bundle branch block, bundle branch reentry, or ventricular activation over an atriofascicular or nodoventricular pathway, are highly unlikely.

Figure 2 demonstrates that an atrial premature depolarization introduced in the right atrium advances the next QRS complex without changing its configuration and terminates the tachycardia. Although it is possible for an atrial premature depolarization to terminate ventricular tachycardia, one generally would expect the QRS complex that results from the atrial premature depolarization to have a different configuration than that of the ventricular tachycardia. An unlikely exception would be an accessory pathway and a coexistent ventricular tachycardia that arises at the ventricular insertion of the accessory pathway. Termination of the tachycardia by an atrial premature depolarization that advances the next QRS complex without changing its configuration is much more likely to indicate either an antidromic reciprocating tachycardia or an atrial tachycardia with conduction over an accessory pathway. The sinus beat present in Figure 2 demonstrates a His-ventricular interval of zero, confirming that an accessory pathway is present. The absence of a His-bundle depolarization preceding the ventricular depolarization in the His-bundle electrogram during the tachycardia suggests that anterograde conduction may be occurring solely through an accessory pathway, thereby favoring antidromic tachycardia over atrial tachycardia; however, the pos- 


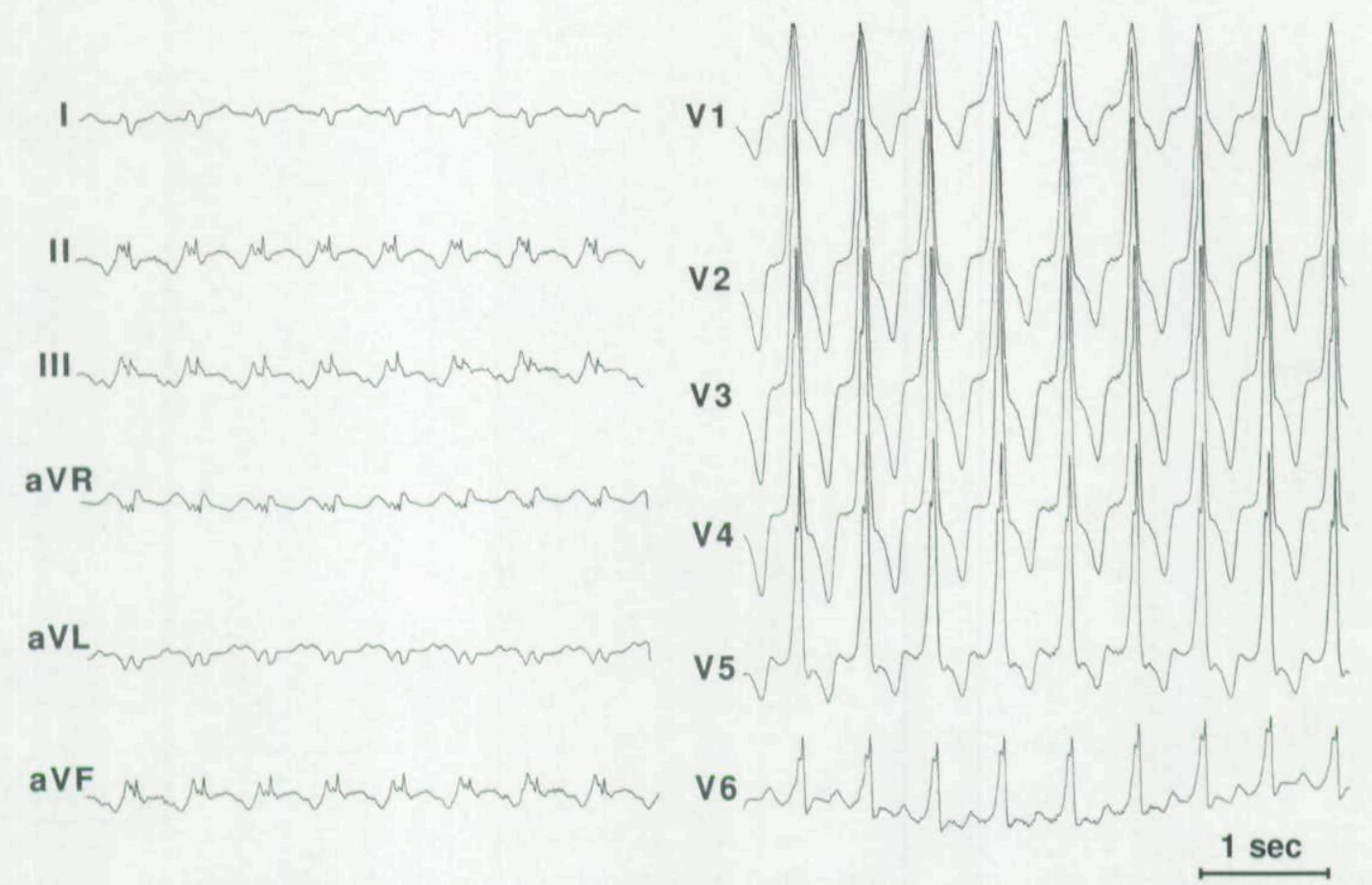

Figure 1. Wide QRS tachycardia, cycle length $540 \mathrm{msec}$. The QRS duration is $180 \mathrm{msec}$.

sibility that this is an atrial tachycardia that conducts through an accessory pathway and also penetrates the AV node, with a long atrial-His interval and a His-bundle depolarization buried in the ventricular electrogram, cannot be ruled out. Therefore, additional evidence is required to distinguish antidromic reciprocating tachycardia from an atrial tachycardia.

The evidence needed to differentiate antidromic reciprocating tachycardia from an atrial tachy-

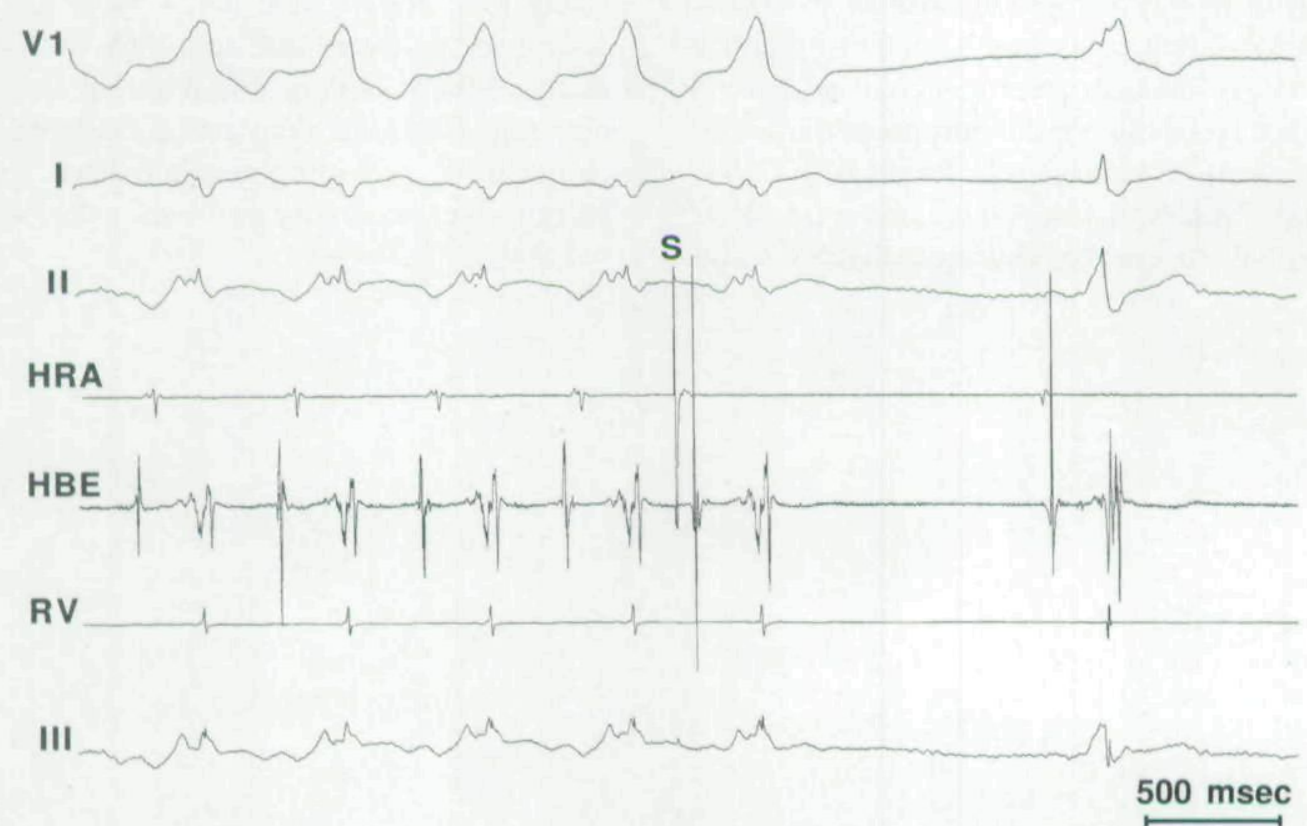

Figure 2. Termination of the tachycardia by an atrial premature stimulus (S). Shown are leads VI, I, and II, high right atrial (HRA) recording, His-bundle electrogram (HBE), right ventricular (RV) electrogram, and lead III. 


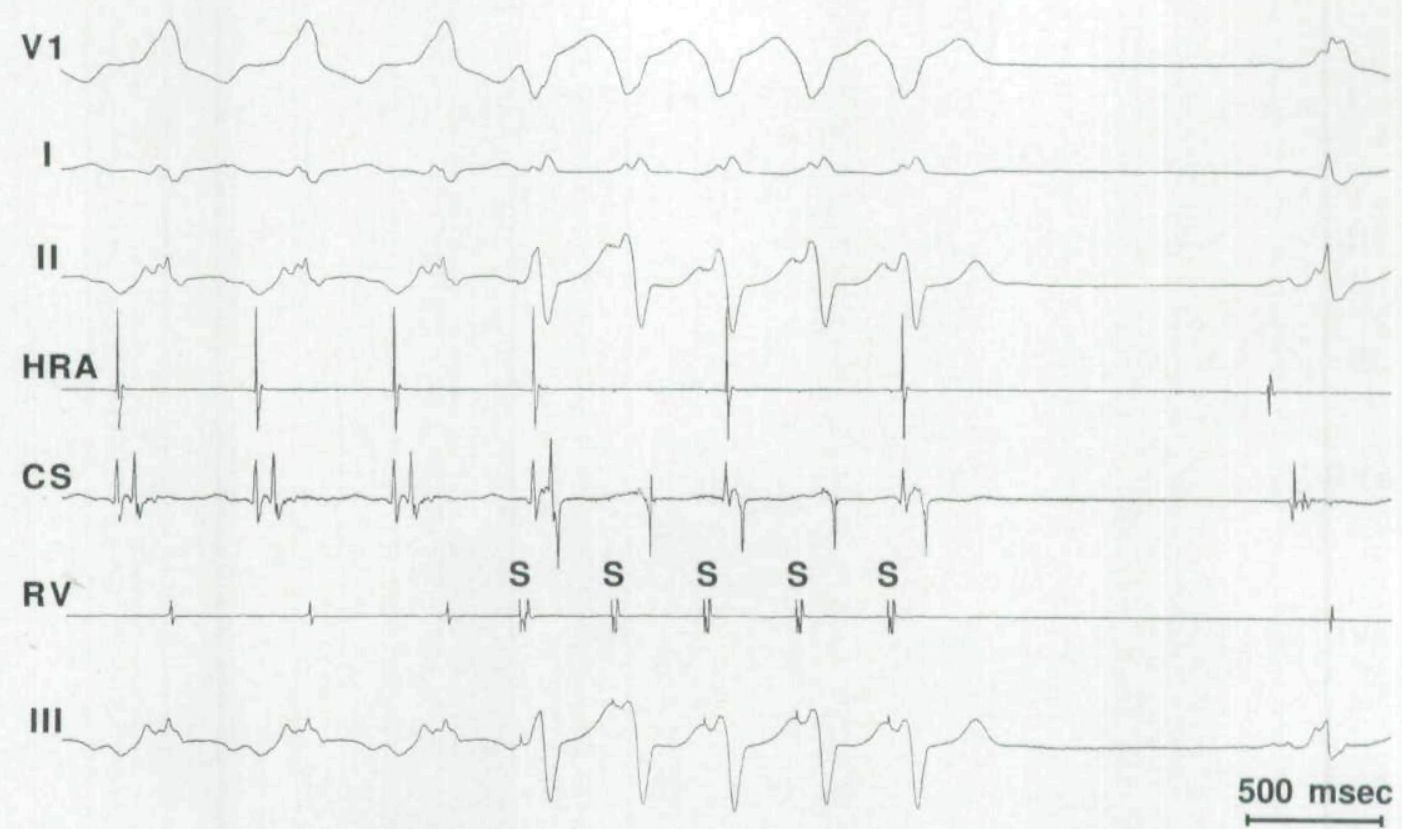

Figure 3. Termination of the tachycardia by right ventricular pacing at a cycle length of $340 \mathrm{msec}$. CS = coronary sinus electrogram recorded adjacent to the lateral aspect of the mitral annulus; $S=$ pacing stimulus.

cardia is present in Figure 3. Ventricular pacing terminates the tachycardia without advancing the atrial electrogram, thereby ruling out an atrial tachycardia.

Although it can be concluded with certainty that the tachycardia is an antidromic reciprocating tachycardia, the information provided does not allow identification of the retrograde limb of the reentry circuit. VA conduction may be occurring either through the AV node or over a second accessory pathway that has slow conduction properties. The pattern of atrial activation was found to be concentric, and a ventricular depolarization introduced in the first half of diastole during tachycardia did not reset the tachycardia, consistent with VA conduction through the AV node.

It was found that the patient had a left lateral accessory pathway with anterograde and retrograde block cycle lengths of 500 and $320 \mathrm{msec}$, respectively. The accessory pathway was successfully ablated with radiofrequency energy. Given the relatively long anterograde block cycle length of the accessory pathway and long VA interval during tachycardia, it is very possible that slow pathway ablation would have prevented maintenance of the tachycardia, providing an alternative approach to therapy had accessory pathway ablation not been feasible. 
This document is a scanned copy of a printed document. No warranty is given about the accuracy of the copy. Users should refer to the original published version of the material. 